\title{
EDITORIAL \\ Reducing the risk of shunt malfunction: beacons of light
}

\author{
Shenandoah Robinson, MD \\ Department of Neurosurgery, Boston Children's Hospital, Harvard Medical School, Boston, Massachusetts
}

$\mathrm{T}$ He recent prospective multicenter study from the Hydrocephalus Clinical Research Network (HCRN) investigators once again provides the field of pediatric neurosurgery with tangible guidance. This time it is for one of our most vexing challenges: how to reduce the risk of ventricular shunt malfunction after initial shunt insertion. For too many years our field has propagated tradition and continued practices based on anecdotal experience rather than solid clinical evidence. While mastering the nuances of surgical technique from mentors will always be important, we also need to incorporate clinical evidence into practice. The new study by Riva-Cambrin and colleagues evaluated 21 factors using a multivariate Cox proportional hazard analysis and found that 3 factors independently predicted an increased risk of shunt malfunction: age younger than 6 months at the time of shunt insertion, endoscope-guided ventricular catheter insertion, and cardiac comorbidity. ${ }^{4}$

In this large, well-designed, and well-executed study, the authors enrolled 1036 children who underwent initial shunt insertion at 6 centers between 2008 and 2011. One strength of this study is that there was a high rate of follow-up, with a mean follow-up interval of 400 days. Only $2.9 \%$ were lost due to out-of-network relocation, and $3.4 \%$ died of causes unrelated to shunt malfunction. A second strength of the study is that the authors decided a priori which factors they would evaluate. Most often in other studies, the factors are selected after the data have been collected. One limitation is that surgical procedures used to treat hydrocephalus prior to permanent shunt insertion, such as an external ventricular drain or endoscopic third ventriculostomy (ETV), were not evaluated.

Overall, one-third of the shunts failed during the study period. The finding that $23 \%$ of the revisions were due to infection is a stark reminder that shunt infection remains a huge hurdle. The shunt infection rate was $7.6 \%$, not that much lower than the $8.1 \%$ present in the Shunt Valve Design Trial from 2 decades ago. ${ }^{2}$ Infection remains a prominent source of morbidity for many patients who have shunt-dependent hydrocephalus, and further investigations of strategies to reduce shunt infections are warranted.

Both from anecdotal experience and from small, singleinstitution studies, young age at the time of insertion and etiology of hydrocephalus have been implicated as risk factors for shunt malfunction. The current study was sufficiently powered to definitively address these issues and demonstrated that the primary culprit is age younger than 6 months rather than etiology of hydrocephalus. While in everyday practice our hand is often forced, leading us to treat hydrocephalus urgently, the information from the current study should encourage us to pause and consider whether temporizing measures might allow postponing definitive surgical treatment until after 6 months of age. Age younger than 6 months was also an independent predictor for failure of ETV combined with choroid plexus coagulation, ${ }^{5}$ which suggests that infants with symptomatic hydrocephalus are particularly difficult to treat. In addition to these neurosurgical studies, emerging evidence suggests that infants, particularly preterm infants, may be at higher risk for loss of cognitive skills from repeated early surgeries and/or anesthesia, although the jury is still out on this complex issue. One must balance the risks of observation with intervention. More well-designed studies in infants are needed to clarify the relative risks of complications such as shunt malfunction and infection to cognition and neurodevelopment.

The current study found the routine use of the endoscope to guide ventricular catheter insertion increased the risk of malfunction. This finding replicates the finding of the Endoscopic Shunt Insertion Trial (ESIT). ${ }^{3}$ Endoscopic fenestration is often effective in reducing the need for mul- 
tiple ventricular catheters in selected cases such as loculated hydrocephalus. Given the results of the current trial and the prior ESIT study, routine use of the endoscope to guide catheter insertion is not supported. Interestingly, use of other guidance adjuncts such as ultrasound and neuronavigation was also not protective in the present study.

An important new finding in this study is the higher incidence of shunt malfunction associated with preexisting cardiac comorbidities. Medical comorbidities were assessed using the complex chronic conditions methodology, which has been validated in pediatric patients. Cardiac comorbidities were defined as heart and great vessel malformations, cardiomyopathies, conduction disorders, and dysrhythmias. These were present in $11.1 \%$ of patients in this series. As the authors note, this risk factor has not been identified in prior studies, except for a recent singlecenter study from 2013 that found an increased risk with of shunt infections associated with prior cardiac surgery. ${ }^{1}$ The emergence of cardiac conditions as a risk factor may be due to improved survival of children with complex cardiac anomalies, or it may be that the risks from other common comorbidities such as gastrostomy tubes no longer pose a higher risk of malfunction. This information is useful in counseling parents.

Perhaps the most important finding from this study is the extensive list of variables that did not minimize the risk of shunt malfunction. Name your favorite risk factor or intervention that has been touted to reduce the chance of shunt malfunction: programmable valves, surgeon experience, antibiotic-impregnated catheters, and a host of others that are often mentioned in the literature or at meetings. Sometimes our intuition is incorrect, such as the thought that endoscopic-assisted catheter placement would be protective. While some factors are not open to change, such as etiology and medical comorbidities, other factors that are controllable should be thoughtfully considered. Some interventions are also associated with significant costs, such as programmable valves and antibiotic-impregnated catheters. For example, if additional prospective, multicenter studies replicate the findings here that antibiotic-impregnated catheters are not protective against malfunction or infection, then perhaps their use should be confined to specific patient populations.

Care should be taken about overextrapolating the current study's findings to one's own hydrocephalus population. Referral patterns differ and the hydrocephalus population of the $6 \mathrm{HCRN}$ centers that participated in this study may not be reflective of other populations. The findings from this study provide guidance and information to counsel parents and medical colleagues where almost no information existed previously, and should be incorporated into practice. There is still a long way to go, especially with the prevention of shunt infections. But the authors have given us some sorely needed illumination along the dark trek to provide enduring, effective treatment for children with hydrocephalus.

http://thejns.org/doi/abs/10.3171/2015.7.PEDS15375

\section{References}

1. Drake JM, Kestle JR, Milner R, Cinalli G, Boop F, Piatt J Jr, et al: Randomized trial of cerebrospinal fluid shunt valve design in pediatric hydrocephalus. Neurosurgery 43:294-305, 1998

2. Kebriaei MA, Shoja MM, Salinas SM, Falkenstrom KL, Sribnick EA, Tubbs RS, et al: Shunt infection in the first year of life. J Neurosurg Pediatr 12:44-48, 2013

3. Kestle JR, Drake JM, Cochrane DD, Milner R, Walker ML, Abbott R III, et al: Lack of benefit of endoscopic ventriculoperitoneal shunt insertion: a multicenter randomized trial. J Neurosurg 98:284-290, 2003

4. Riva-Cambrin J, Kestle JRW, Holubkov R, Butler J, Kulkarni AV, Drake J, et al: Risk factors for shunt malfunction in pediatric hydrocephalus: a multicenter prospective cohort study. J Neurosurg Pediatr [epub ahead of print December 4, 2015. DOI: 10.3171/2015.6.PEDS14670]

5. Stone SS, Warf BC: Combined endoscopic third ventriculostomy and choroid plexus cauterization as primary treatment for infant hydrocephalus: a prospective North American series. J Neurosurg Pediatr 14:439-446, 2014

\section{Disclosures}

The authors report no conflict of interest.

\section{Response}

\section{Jay Riva-Cambrin, MD, MSc, ${ }^{1}$ John R. W. Kestle, MD, ${ }^{2}$ and Tamara D. Simon, MD, MSPH, ${ }^{3}$ for the Hydrocephalus Clinical Research Network}

1Department of Neurosciences, University of Calgary, Calgary, Alberta, Canada; 'Department of Neurosurgery, Primary Children's Hospital, University of Utah, Salt Lake City, Utah; and ${ }^{3}$ Department of Pediatrics, University of Washington/Seattle Children's Hospital, Seattle, Washington

We in the HCRN greatly appreciate the comments made by Dr. Robinson in regard to the network as a whole but especially considering the present paper.

Dr. Robinson adeptly noted that shunt infection continues to plague pediatric hydrocephalus patients as shunt infection rates have only mildly diminished over the last 2 decades. We would refer the readers to our previous work by Simon et al. ${ }^{1}$ specifically examining risk factors for shunt infection in this population. Interestingly, the HCRN found that other than previous neurosurgery, age, and the presence of a gastrostomy tube, the highest independent association with shunt infection was shunt revision with a hazard ratio of 3.9 with a single revision and 13.0 with 2 or more. This underscored the importance of pediatric neurosurgeons focusing clinically and in research settings on minimizing the need for shunt revisions in order to meaningfully diminish shunt infections.

The other very salient point Dr. Robinson emphasized was whether the HCRN's component centers and therefore results are generalizable to all pediatric neurosurgery practices. Admittedly, all 6 participating centers in this study are academic and represent moderate- to highvolume tertiary care centers. However, there is considerable variation in hydrocephalus volume between HCRN centers, with a wide geographic representation across 2 countries and therefore health care systems.

In 2009, the HCRN conducted an unpublished internal validation examining the Core Data Project or Hydro- 
TABLE 1. Comparing patient characteristics between the HCRN and the PHIS

\begin{tabular}{|c|c|c|}
\hline Variable & HCRN (\%) & PHIS (\%) \\
\hline No. of patients & 678 & 5559 \\
\hline \multicolumn{3}{|l|}{ Patient age (yrs) } \\
\hline$<1$ & $195(28.8)$ & 31.8 \\
\hline $1-2$ & $103(15.2)$ & 13.5 \\
\hline $3-4$ & $53(7.8)$ & 8.3 \\
\hline $5-10$ & $136(20.1)$ & 20.9 \\
\hline $11-16$ & $154(22.7)$ & 21.0 \\
\hline$>16$ & $37(5.5)$ & 4.4 \\
\hline \multicolumn{3}{|l|}{ Sex } \\
\hline Male & $397(58.6)$ & 56.7 \\
\hline Female & $281(41.4)$ & 43.3 \\
\hline \multicolumn{3}{|l|}{ Race/ethnicity } \\
\hline Black, non-Hispanic & $94(13.9)$ & 17.2 \\
\hline White, non-Hispanic & $335(49.4)$ & 53.7 \\
\hline Hispanic & $107(15.8)$ & 16.9 \\
\hline Asian/Pacific Islander & $8(1.2)$ & 1.4 \\
\hline American Indian & $1(0.1)$ & 0.5 \\
\hline Other & $98(14.5)$ & 10.3 \\
\hline \multicolumn{3}{|l|}{ Payer } \\
\hline Private & $225(37.6)$ & 32.6 \\
\hline Public/government & $364(53.7)$ & 49.2 \\
\hline Self-pay & $17(2.5)$ & 1.5 \\
\hline Other & $9(1.3)$ & 16.0 \\
\hline Missing & $0(0)$ & 0.7 \\
\hline \multicolumn{3}{|c|}{ CCC (excluding hydrocephalus) } \\
\hline 0 & $423(62.4)$ & 42.7 \\
\hline 1 & $199(29.4)$ & 36.7 \\
\hline$\geq 2$ & $56(8.3)$ & 20.6 \\
\hline
\end{tabular}

$\mathrm{CCC}=$ complex chronic conditions .

cephalus Registry in its infancy. We obtained institutional review board/research ethics board approval at all the centers and grossly compared our sample to that of the Pediatric Health Information System (PHIS) database. PHIS is run by the Children's Hospital Association and represented 41 pediatric hospitals contributing data, including 2 of the 4 HCRN centers. At that time, the HCRN consisted of 4 centers and the Core Data Project had 678 patients enrolled, representing 988 hydrocephalus procedures, compared with 5559 patients and 6536 procedures in the PHIS. This confirmed that the HCRN did represent higher volume centers, as volume was an original selection criterion for HCRN membership. However, in terms of patient mix, we found no significant differences in patient age,
TABLE 2. Comparing neurosurgical CSF diversion procedures between the HCRN and the PHIS

\begin{tabular}{lcccc}
\hline \multicolumn{1}{c}{ Procedure } & $\begin{array}{c}\text { HCRN } \\
\text { (no.) }\end{array}$ & $\begin{array}{c}\text { HCRN } \\
(\%)\end{array}$ & $\begin{array}{c}\text { PHIS } \\
\text { (no.) }\end{array}$ & $\begin{array}{c}\text { PHIS } \\
(\%)\end{array}$ \\
\hline Total no. of procedures & \multicolumn{2}{c}{988} & \multicolumn{2}{c}{6536} \\
\hline Initial CSF shunt & 237 & 24.0 & 1721 & 26.3 \\
\hline Revision & 609 & 61.6 & 4008 & 61.3 \\
\hline Infection procedures & 142 & 14.3 & 807 & 12.3 \\
$\quad$ Removal/externalization & 74 & 7.5 & NA & NA \\
$\quad$ Reimplantation & 68 & 6.9 & NA & NA \\
\hline Initial ETV & 40 & 3.8 & NA & NA \\
\hline Revision ETV & 11 & 1.0 & NA & NA \\
\hline Ventricular reservoir & 10 & 0.9 & NA & NA \\
\hline Subgaleal shunt & 11 & 1.0 & NA & NA \\
\hline
\end{tabular}

$\mathrm{NA}=$ not available in PHIS

sex, race/ethnicity, or payer between the HCRN and PHIS, which proposes to represent the North American sample (Table 1). We did find that the PHIS sample had higher rates of comorbidities (Table 1), which was probably a result of the PHIS method of having hospital coders code morbidities following admission, whereas HCRN coding is performed in real time by specific HCRN data coordinators on the date of surgery.

In terms of procedures, Table 2 reveals the difficulty of identifying ETV procedures by the PHIS method of using procedure codes, in contrast to the the accurate, real-time procedure identification method of the HCRN. However, the central theme of Table 2 is that the overall breakdown of hydrocephalus procedures is nearly identical when comparing the HCRN and PHIS (i.e., 61.3\% of procedures in the HCRN data are shunt revisions vs $61.6 \%$ in the PHIS sample).

Therefore, HCRN demonstrated in early internal unpublished work that, despite representing higher volume centers in general, our patient sample and hydrocephalus case mix were very consistent with the PHIS database and therefore with the (North) American population in general. For this reason, we believe that most HCRN studies and results thereof are generalizable to the majority of pediatric neurosurgery practices.

\section{References}

1. Simon TD, Butler J, Whitlock KB, Browd SR, Holubkov $\mathrm{R}$, Kestle JR, et al: Risk factors for first cerebrospinal fluid shunt infection: findings from a multi-center prospective cohort study. J Pediatr 164:1462-1468, 1468.e1-1468.e2, 2014 